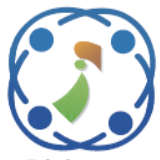

\title{
An Efficient Algorithm for End-to-End Latency Optimization Over IEEE 802.15.4 Wireless Network for IoT Applications
}

\author{
Imane Dbibih $^{1 *} \quad$ Imad Iala $^{2} \quad$ Fatima Zahra El Biach $^{2} \quad$ Ouadoudi Zytoune $^{3}$ \\ ${ }^{1}$ Laboratory of Engineering Sciences and Energy, Polydisciplinary Faculty of Ouarzazate, \\ Ibn Zohr University, AGADIR, Morocco \\ ${ }^{2}$ LRIT Associated Unit to the CNRST-URAC N29, Faculty of Sciences, University Mohammed V, Rabat, Morocco \\ ${ }^{3}$ ENSA, University Ibn tofail Kenitra, Morocco \\ * Corresponding author’s Email: i.dbibih@gmail.com
}

\begin{abstract}
The continuous evolution of new wireless technologies has given birth to new application areas for the Internet of Things (IoT) such as Smart Cities, Smart Home, Intelligent Transport Systems, Wireless Sensor Networks, etc. IoT is a network of networks that enables, via small wireless electronic devices called "sensor", to identify and communicate with physical objects. The main IoT objective is to measure and exchange data between physical and virtual worlds. However, the use of these sensors in applications characterized by the coexistence of different traffic loads in the same network could lead to power consumption and latency problems. In this article, we present a new efficient algorithm called (BMPriority-based CSMA/CA) based on message priority and battery energy level of sensor to manage access to the transmission channel. More precisely, our algorithm uses a weighting function to calculate the contention window during which a sensor node must wait before starting its data transmission. Simulation results show that our proposed protocol outperforms S-MAC, IEEE 802.15.4, and ECA-MAC protocols in terms of optimizing Packet Delivery Ratio (PDR), end-to-end latency, and throughput.
\end{abstract}

Keywords: IoT, Mac protocol, Energy priority, Message priority, Services differentiation, Heterogeneous sensing.

\section{Introduction}

Nowadays, the large-scale diffusion of the Internet enables devices and smart objects to communicate, cooperate, and make decisions. This promising system is known as the "Internet of Things" (IoT) and its evolution goes with the progress of the new communication technologies, such as RFID, Wireless Sensor Networks (WSNs), wearable sensors, actuators, etc.[1].

In WSN-based IoT applications, devises can be deployed for monitoring different phenomena, and therefore generate heterogeneous traffic in the network [2]. Usually in this kind of applications, there is heterogeneity in terms of either traffic loads or the nodes capacities. To resolve the related problems of heterogeneous WSN-based IoT applications, we need to design new communication protocols able to differentiate services. The MAC layer considered as one of the key layers that can provide good QoS in WSNs. However, the most of MAC protocols, designed for them, take into account energy limitation of nodes at the expense of the other performance criteria.

6LoWPAN is a technology [3] that offers a proprietary and lightweight protocol stack. It is based on the IEEE 802.15.4 [4] standard for the physical and MAC layers and offers its own upper layers (network, etc.); 6LoWPAN allows the transport of IPv6 packets via the IEEE 802.15.4 standard. This latter standard is heavily used in WSN applications; it performs low energy consumption due to dutycycled operation, which alternates the radio between inactive and active states. In the coordinated mode, there are two medium access periods during the active time; CAP (Contention access period) and CFP (Contention free period). In the first period, all nodes get access the medium based on slotted CSMA/CA algorithm. However, during the second one, the 


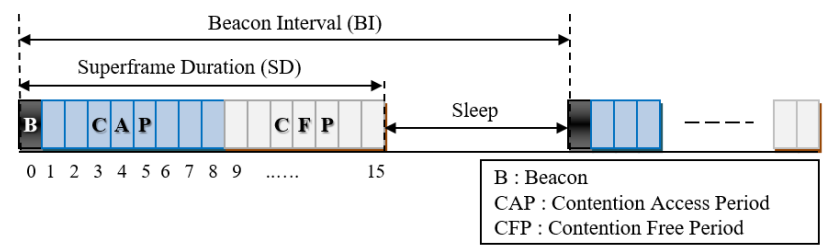

Figure. 1 IEEE 802.15.4 super-frame structure in beaconenabled mode

coordinator guarantees to devices a specified number of slots for free medium access. CFP period offers Guarantee Time Slots (GTS) to nodes having realtime traffic (see Fig. 2).

The works [5-11] try to analyse the performance of both CAP and CFP periods; they all conclude that the parameter values used by the standard adversely affect the performance of sensor networks. In order to create a new algorithm that is able to satisfy the heterogeneous WSN requirements we propose, in this paper, to manage the quality of service during the CAP period of IEEE 802.15.4 super-frame. We propose a new scheme based on the slotted CSMA/CA algorithm, which considers two priority criteria. The first criterion concerns the message priority in order to reduce the latency of real-time traffic. The second one concerns the battery lifetime of the transmitter node, which aims to improve nodes implication in the network. This second priority allows to a node having a critical battery lifetime, to get more chance to access the channel for sending its data before it runs out.

The remainder of this paper is organized as follows. Section 2 presents a summary of works that have enhanced the standard in terms of QoS. Section 3 details our contribution. Then, the performance analysis and the experiment results are shown in Section 4, while Section 5 summarizes the paper conclusion with referred prospects.

\section{Related works}

Several studies have been published in order to analyse the performance of IEEE 802.15.4 standard, confirm all the poor performances (latency, Packet Delivery Ratio, throughput) in a large-scale network. While other works, such as [5, 7-11] attempt to evaluate the impact of the slotted CSMA/CA parameters over the performance criteria of WSNs. According to Anastasi et al. [5], the standard provides a very low level of PDR (Packet Delivery Ratio) due to the parameters defined by the standard for the slotted CSMA/CA algorithm. While in [6] and [7] the authors proved through experiments that the high values of macMinBE maximize the probability that the message reaches its destination in the first attempt.
In three studies $[5,8,9]$ the authors confirm that the performance criteria (PDR, throughput) of IEEE 802.15.4 networks are poor either in multi-hop or star topology. These results are due to the value of $B E$, which does not effectively manage the medium access when there are a large number of nodes deployed in the network.

The analyses carried out confirm that the standard does not have mechanisms to efficiently manage node priority to access the medium. Many works have tried to process the concept of priority for heterogeneous applications. To differentiate the medium access priority, Boughanmi et al. [10] have used a new mechanism based on CSMA/CA algorithm called Blackburst message. When a node wants to access the medium, it waits for a time interval called LIFS (long inter-frame space) before it sends its Blackburst message, which its length depends on data priority. The problem is that the transmission of the Blackburst message will increase energy consumption. As well, this method will influence negatively the latency, due to the wait time of LIFS and $T_{o b s}$ periods.

For the same purpose, in [13] authors propose a new period at the beginning of each super-frame called PAP (Priority Access Period) assigned by the coordinator. Its objective is to provide an opportunity for a node to send its real-time data or asking for guaranteed time slots from the coordinator. The disadvantage of this method is that the PAP period is not allocated according to the priority of nodes traffic; therefore, it does not improve the QoS of the highest priority messages.

While in ([11, 14, 15]), authors propose to classify the traffic according to its priority to provide a quality of service to sensor networks. In [11], the authors handle the medium access according to the node priority. According to each level, we can set the minimum and the maximum of the backoff exponent (macMinBE, aMaxBE repectively) and the contention window $(C W)$. The results show the effectiveness of this method in terms of throughput/workload. However, the major drawback is that this scheme is based on a deterministic topology, which is not always realistic even if there are a low number of nodes deployed in the network.

Also, in [14] the authors create an analytic model of medium access contention, during the CAP period of IEEE 802.15.4. This study was carried out to evaluate the effect of the $C W$ parameter on the medium contention of nodes having prioritized messages. In this work, the authors confirm through simulations the efficiency of $\mathrm{CW}$-based service differentiation. However, this study is tested using a non-large scale network, they considered a start- 
topology with a small number of nodes (only 12 nodes) which is not realistic for sensor networks. To get the same purpose, [15] proposes a new CSMA/CA-based scheme called MP-based CSMA/CA that considers two priority levels; high priority assigned to critical-time messages and low priority assigned to non-real-time messages. For each one of both types, authors define a different contention window. The problem with this method is that the nodes having low priority messages are forced to wait until the second window even there is no high priority messages to relay.

In literature, there were many contributions [16, 17, 18], and others tackling IEEE 802.15.4 performances by improving the CFP period. In [16], the proposed protocol OGMAD tries to adjust efficiently the length of CFP according to GTS requests received from network nodes. However, in general, CFP-based methods are not serving the medium access priority. The GTS requests are sent to the coordinator during the CAP period, when nodes are using slotted CSMA/CA. This last is not considering medium access priority, which means that we can't guarantee priority access during CFP. Also, Gupta et al. [17] propose a new scheme based on GTS allocation. More GTSs are dynamically allocated to the node having an abrupt increase in data rate. However, in this way, we risk starving other nodes of their rights to get a GTS. In [18], the authors propose a new MAC protocol targeting QoS, by attributing a static priority to sources and intermediate nodes, which is not matching with the nature of WSN that are AD-HOC networks, where nodes are deployed randomly.

On the other hand, many studies addressed the WSN problems and proposing solutions that aim at reducing the energy dissipation and the end-to-end latency due to idle listening problem. These solutions $[23,24]$ are based on the duty-cycle scheme principle to extend the network lifetime by optimizing the transceiver energy consumption without influencing negatively the latency. The authors of [25] proposed an algorithm called Energy based Collision Avoidance (ECA-MAC) that aims to control the access to the medium according to nodes energy level and to reduce the collision by the use of several Contention Windows. In this work, the authors only used the energy level to prioritize the access to the channel, while this method does not take into account the priority of the messages, unlike our proposed solution, which makes it possible to combine the two priorities (energy level and messages priority) to promote access to the transmission channel.

None of the cited works ever handled the priority to get the medium based on the node's energy level and message priority. Taking into consideration the both criteria in the provided chance to get access to the medium is the point that makes the difference between our protocols and others. Counting the energy level can considerably help to improve the network performances, as we will see in section 5. In our work, we consider two important criteria to handle medium access. In addition to the priority of the message, we also take into consideration the energy status of the relaying node. The more the node is in critical energy level, the more it has the chance to get the medium and send its messages before running out.

\section{The proposed approach}

BMPriority-based CSMA/CA algorithm proposes to enhance the performances of deployed WSNs for IoT applications. Therefore, it intends to improve the quality of service in terms of prioritized messages latency, to increase the throughput and to manage the good implication of nodes in the network. In this paper, we aim to get better the medium access scheme during the CAP period of the IEEE 802.15.4 standard, in which nodes access medium by contention. In our contribution we extend the CAP period along the active time; According to the authors $[19,20]$, the size of the slots allocated to one node may exceed its transmission needs, which leads to a bandwidth wastage. Also, in [21] authors confirm that this period is not adapted to the needs of realtime messages.

Our algorithm gives more chance to nodes, to gain medium access contention than other nodes, based on two priority criteria. The first one is based on the message priority, while the second one is based on the battery energy-level priority of the node. It is known that in WSNs nodes are powered by irreplaceable battery. Therefore, to properly use energy resources, we give to a node that has a critical battery energy level a high priority to access the channel and to transmit its data before that its battery runs out.

\subsection{Battery and message priority-based CSMA/CA algorithm}

The process of our algorithm consists of two phases; the first concerns the priority assignment to the nodes and calculating a global priority, whereas the second concerns the medium access mechanism based on the calculated global priority.

To calculate the Global Priority GP we need two parameters; MsgPriority (Message Priority) that depends on the type of the sent messages and EnrgPriority (Energy Priority) that is defined 
according to the battery energy level. We consider that the assignment of the value of MsgPriority parameter is accorded to one of the upper layer, based on the message nature. While, EnrgPriority gets its priority at the MAC layer based on the status of the battery power-level. For both priorities, we differentiate three priority levels. The parameter MsgPriority takes the value 1 if the sent message has the highest priority (assigned to real-time messages; that require very low transmission delay) and takes 2 or 3 if the sent message has medium or low priority respectively. As well, the EnrgPriority parameter allows us to specify the priority given to a node based on its current battery power. We have defined three levels, on which a node's current battery power can belong (see Eq. 1). Thus, for each level, EnrgPriority parameter has its own priority value. The first level is the one that is below the threshold $S_{1}$ (is one-third of the initial battery power). In this case, the node is in a critical situation and it can run out at any time. For this level of battery power, the node gets the highest priority, and thus the value 1 is accorded to the EnrgPriority parameter. The second level is the one that is included between $\mathrm{S}_{1}$ and $S_{2}$ (is two-thirds of the initial battery power). At this level, the node gets a medium priority, and thus EnrgPriority $=2$. The third level is the one that is above the threshold $\mathrm{S}_{2}$. In this case, the node has a good battery power level that can guarantee a longer lifetime compared to the other levels, so the node is not in a critical situation in terms of battery. Consequently, the node gets the lowest priority, and thus EnrgPriority $=3$. Both thresholds $S_{1}$ and $S_{2}$ are defined following the initial power (InitialEnergy) of each node's battery.

$$
\left\{\begin{array}{c}
S_{1}=\frac{\text { InitialEnergy }}{3} \\
S_{2}=\frac{\text { InitialEnergy } \times 2}{3}
\end{array}\right.
$$

As reported previously, the privilege of accessing the medium depends on two priority criteria (MsgPriority, EnrgPriorit). Accordingly, we calculate the global priority $(G P)$, taking into account the value of each priority following Eq.2:

$$
\begin{array}{r}
G P=\alpha \times \text { MsgPriority }+(1-\alpha) \times \\
\text { EnrgPriority }
\end{array}
$$

Knowing that $\alpha$ and $1-\alpha$ are the multiplicative factors associated to MsgPriority and EnrgPriority respectively. $\alpha$ allows us to adjust the weight of each one of both parameters,
Table 1. Values of GP and BE for $\alpha=0.3$

\begin{tabular}{|c|c|c|c|}
\hline MsgPriority & EnrgPriority & $\boldsymbol{G P}$ & $\boldsymbol{B E}$ \\
\hline 1 & 1 & 1.0 & 2 \\
\hline 2 & 1 & 1.3 & 3 \\
\hline 3 & 1 & 1.6 & 4 \\
\hline 1 & 2 & 1.7 & 5 \\
\hline 2 & 2 & 2.0 & 6 \\
\hline 3 & 2 & 2.3 & 7 \\
\hline 1 & 3 & 2.4 & 8 \\
\hline 2 & 3 & 2.7 & 9 \\
\hline 3 & 3 & 3.0 & 10 \\
\hline
\end{tabular}

MsgPriority and EnrgPriority, on the global priority. The multiplicative factor $\alpha$ is a real number in the range $[0,1]$. More its value is close to 1 , more MsgPriority parameter is favoured over EnrgPriority parameter, and vice versa. Thus, the choice to strengthen one priority over the other depends on application needs.

In the second phase, our algorithm deals with service differentiation problem by according to each priority level a specific backoff range to get access the medium. It intends to manage the medium access according to the calculated global priority $(G P)$, that has a real value in the range [1,3]. According GP's value we adjust $B E$ (Backoff Exponent) so that it gets an integer number between 2 and 10. $B E$ parameter allows us to define how many BP (Backoff Period, the smallest unit of time) a device shall wait before attempting to access the channel (see Eq. 3).

After calculating $G P$, we classify its value into the range $[1,3]$ and then based on its classification, $B E$ takes linearly one value that belong to the range $[2$, $10]$. $B E$ depends on $G P$; more we have a low value of the global priority $G P$, more the value of $B E$ is low in the range $[2,10]$. To more understand this process, see the presented example in Table. 2 that illustrates all the values of $G P$ and $B E$ for $\alpha=0.3$.

Once $B E$ is adjusted, the node can calculate the time to wait(Wtime), before trying to get access the medium. Wtime is a random number of $\mathrm{BP}$ in the range of 0 to $\mathrm{BE}$ (see Eq.3).

$$
\text { Wtime }=\operatorname{random}([0, B E]) \times B P
$$

In case of collisions, Slotted CSMA/CA algorithm[4]increases exponentially the wait time range, which causes a high latency. To cope with this problem and reduce latency due to collisions, our algorithm proposes to linearly increase $\mathrm{BE}$ as well as Wtime (see Fig. 2).

In our algorithm, nodes have different ranges to take their random values. For example (for $\alpha=0.3$ ), 


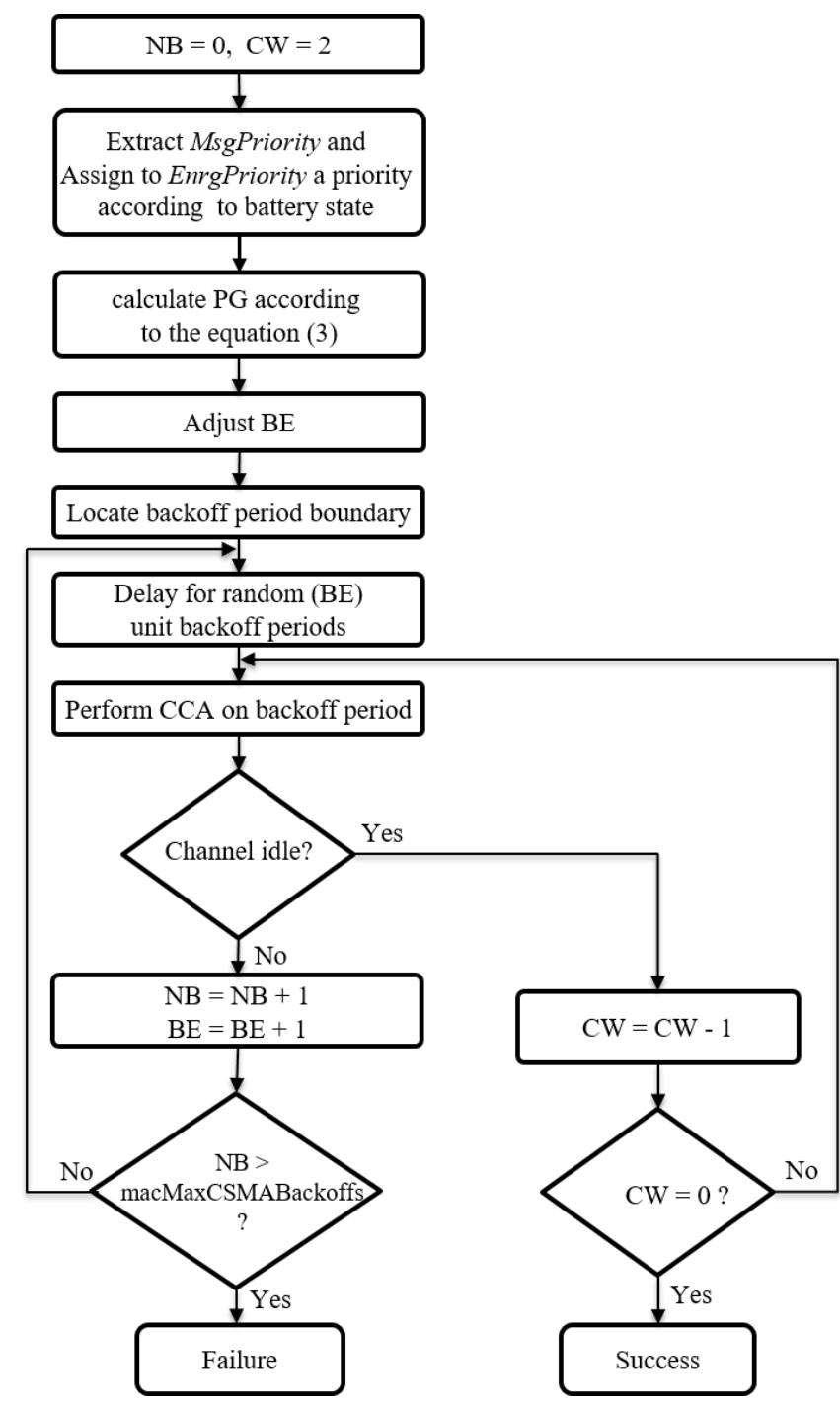

Figure. 2 Battery and Message Priority-based CSMA/CA algorithm

if two nodes want to access the medium $N_{1}(G P=$ 1.3) and $N_{2}(G P=2.3)$ (see Table.3), the node that has the highest priority, $N_{1}$, will obtain a lower value of $B E$ that is 3. So, according to Eq.3, $N_{1}$ will automatically take a small value ofWtime. However, for the other node $N_{2}$, it is not necessarily that it takes a lower value of $W$ time because the range of random values of $N_{2}([0,7])$ is wider than that of the first node $N_{1}$. Moreover, since the used random function in our implementation follows a uniform distribution, so the chance to take a small random value is $\frac{1}{3}$ for $N_{1}$, however, for $N_{2}$ is only $\frac{1}{7}$. In this way, the node $N_{1}$ has more chance to get access the channel before $N_{2}$, and transmit its data in the first attempt.

Fig. 2 shows the process of the BMPriority-based CSMA/CA algorithm. It consists of six steps:

- Step (1): initialize the parameters $\boldsymbol{N B}=\mathbf{0}$ and $\boldsymbol{C W}=2$.
- Step (2): extract the MsgPriority, assign a priority to EnrgPriority according to the battery energy level, calculate the global priority $G P$ according to Eq.2 and adjust $B E$ according to the calculated $G P$.

- Step (3): calculate the Wtime using (Eq.3).

- Step (4): after the expiration of the wait time, performs CCA, to assess whether the channel is idle. If the channel is assessed to be busy the node goes to Step (5), otherwise, it goes to Step (6).

- Step (5): if the channel is busy, the values of $N B$ and $B E$ are increased by one. Then the node tests if the value of $N B$ exceeds macMaxCSMABackoffs, if this is the case it declares a transmission failure. Otherwise, it goes to Step (3).

- Step (6): if the channel is not busy, the value of $C W$ is decreased by one, and then the node tests whether $C W$ reaches 0 , if this is the case it access the channel, otherwise it goes to Step (4).

\subsection{A network scenario in which nodes use BMPriority-based CSMA/CA algorithm}

In this example, we will show the difference that can make the choice of the multiplicative factor $\alpha$ on the performance of the application.

We suppose that there are three nodes $N_{1}, N_{2}$ and $N_{3}$ in the same transmission range, and these nodes want to access the medium at the same time, but each one has different values of MsgPriority and EnrgPriority (see Fig. 3). Firstly, we should specify the multiplicative factor; we suppose in the first scenario that $\alpha=0.2$. In this case, we give more importance to the battery energy-level priority. According to the combinations (MsgPriority, EnrgPriority)(given in Fig. 3), we calculate GP and adjust $B E$ of each node:

$$
\left\{\begin{array}{l}
N_{1}: G P=0.2 \times 1+0.8 \times 3=2.6 \rightarrow B E=8 \\
N_{2}: G P=0.2 \times 2+0.8 \times 2=2 \rightarrow B E=6 \\
N_{3}: G P=0.2 \times 3+0.8 \times 1=1.4 \rightarrow B E=4
\end{array}\right.
$$

In this case, it is $N_{3}$ that has the highest priority compared of the other nodes (lower value of $(G P)$ ). Thus, it obtains a lower value of $B E$, and therefore the greatest chance to gain the medium access contention.

We consider a second scenario where the battery energy level is less important than the message priority. According to these needs, $\alpha$ can be set at 0.7 . So, the global priorities and $B E$ calculated as follow:

$$
\left\{\begin{array}{l}
N_{1}: G P=0.7 \times 1+0.3 \times 3=1.6 \rightarrow B E=4 \\
N_{2}: G P=0.7 \times 2+0.3 \times 2=2 \rightarrow B E=6 \\
N_{3}: G P=0.7 \times 3+0.3 \times 1=2.4 \rightarrow B E=8
\end{array}\right.
$$




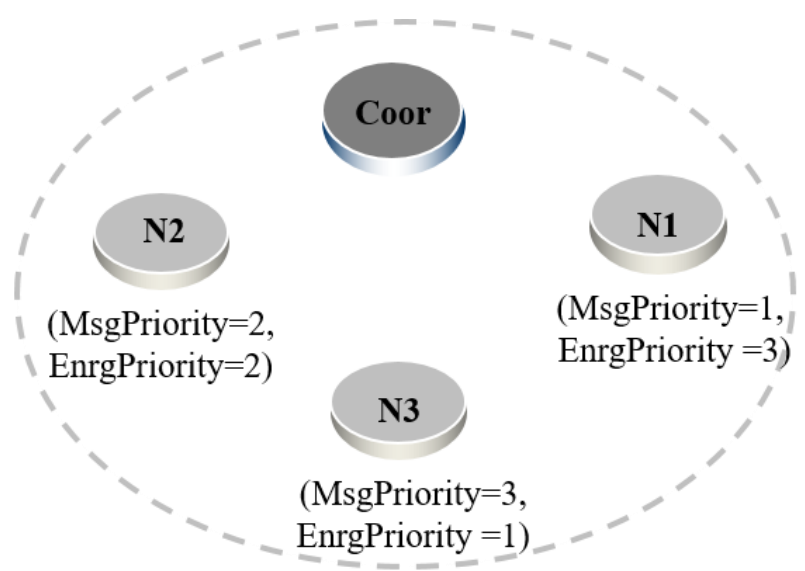

Figure. 3 Example of scenario for three nodes accessing the medium with a coordinator

In this case, it is $N_{1}$ that has the highest priority, which allows it to access the medium with a high probability.

In the first case, it is $N_{3}$ which has the highest battery energy-level priority. Therefore, according to our algorithm, it has a high probability of accessing the medium. However, in the second case, it is $N_{1}$ which has the greatest chance to win the contention access to the channel, because it has the highest message priority.

\section{Simulation results}

In this section, we carry out several simulations to demonstrate BMPriority-based CSMA/CA algorithm. We prove the effectiveness of our algorithm by evaluating, on the first hand, the network performance metrics (latency, PDR, and throughput) under several simulation scenarios, on the other hand, by comparing the obtained results by our algorithm with those obtained by the IEEE 802.15.4 standard [4], SMAC [26] protocol and ACE-MAC algorithm [25].

\subsection{Performance criteria}

BMPriority-based CSMA/CA algorithm aims to improve the performances of heterogeneous WSNbased IoT, in which nodes having real-time messages or high-prioritized data must obtain a great chance to access the channel. Accordingly, we need that once the prioritized message gets to any intermediate node (along the transmission path) this latter wins the medium access contention. Therefore, to prove the efficiency of our algorithm in this point, we evaluate the latency of the prioritized messages. On other hand, our algorithm tries to handle the medium access priority according to the battery energy level of the node. The objective is to offer to nodes having a critical battery energy level, more chance to access the medium, and then it can transmit the maximum of messages in its queue. To show the effectiveness of our contribution, we calculate the number of sent packets when the energy level is lower than the first threshold $S_{1}$ (see Eq. (1)). As well, we evaluate the basic performance criteria (throughput, latency of all packets transmitted in the network and PDR).

\section{Simulation parameters}

Our solution was implemented and tested using the model, denoted as WPAN, in NS-2 (Network simulator) [22]. This latter is well known and it is among of the most used simulators to study the performances of network protocols. In these simulations, we compare our algorithm with the standard IEEE 802.15.4, and since we do not consider CFP period in our algorithm, so we will extend the CAP period along the active time also for the standard.

In this section, we present a comparison between BMPriority-based CSMA/CA algorithm results and those of the standard IEEE 802.15.4 [4]. We set up our simulations in the Beacon-enabled mode. In these simulations, we evaluate the performance of our solution in mesh topology. The number of nodes deployed in the network is fixed to 100 nodes, and we increase linearly the source nodes (nodes generating the traffic in the network) from 10 up to 100 nodes, in order to vary the traffic load in the network. At the application layer we used the CBR traffic (Constant Bit Rate) produced by source nodes, which generate packets with 100 Bytes $/ \mathrm{s}$. The value of system parameter settings for our algorithm and the slotted CSMA/CA algorithm are cited in Table.2.

The nodes are randomly deployed in an area of $300 \times 300 \mathrm{~m}$. We use one PAN (personal area network) coordinator, and all other nodes are devices. We choose to simulate in this area (to create a multihope transmission) in order to evaluate the effectiveness of BMPriority-based CSMA/CA algorithm on the behavior of the intermediate nodes. The priority of the message provides to nodes more chance to obtain the medium access, which will reduce latency along the path between the source and the destination. In addition, the energy level is lower in the intermediate nodes so that increase the chance to gain the medium contention, and forward the prioritized messages. The results illustrated in the following figures (Fig.6 to 12) present the average of 10 simulations.

In order to show our algorithm efficiency in terms of energy priority, we vary the initial energy linearly 
Table 2. Simulation parameters in NS-2

\begin{tabular}{|c|c|c|}
\hline Parameter & $\begin{array}{c}\text { BMPriority } \\
\text { Protocol }\end{array}$ & $\begin{array}{c}\text { Slotted } \\
\text { CSMA/CA }\end{array}$ \\
\hline BO & 4 & 4 \\
\hline SO & 3 & 3 \\
\hline macMinBE & $2-10$ & 3 \\
\hline aMaxBe & $6-14$ & 5 \\
\hline Duty-cycle & \multicolumn{2}{|c|}{$50 \%$} \\
\hline Beacon interval & \multicolumn{2}{|c|}{$0.24576 \mathrm{~s}$} \\
\hline Active period & \multicolumn{2}{|c|}{$0.12888 \mathrm{~s}$} \\
\hline Inactive period & \multicolumn{2}{|c|}{$0.12888 \mathrm{~s}$} \\
\hline Space range & \multicolumn{2}{|c|}{$300 \times 300 \mathrm{~m}$} \\
\hline Transmission range & \multicolumn{2}{|c|}{$150 \mathrm{~m}$} \\
\hline Simulation time & \multicolumn{2}{|c|}{$800 \mathrm{~s}$} \\
\hline Number of nodes & \multicolumn{2}{|c|}{100} \\
\hline $\begin{array}{l}\text { Number of source } \\
\text { nodes }\end{array}$ & \multicolumn{2}{|c|}{$10-100$} \\
\hline Type of topology & \multicolumn{2}{|c|}{ Mesh } \\
\hline Routing protocol & \multicolumn{2}{|c|}{ AODV } \\
\hline Application layer & \multicolumn{2}{|c|}{ CBR } \\
\hline Data packet size & \multicolumn{2}{|c|}{100 Bytes } \\
\hline Transmit power & \multicolumn{2}{|c|}{$36 \mathrm{Mw}$} \\
\hline Receive power & \multicolumn{2}{|c|}{$14.4 \mathrm{~mW}$} \\
\hline Idle power & \multicolumn{2}{|c|}{$14.4 \mathrm{~mW}$} \\
\hline Sleep power & \multicolumn{2}{|c|}{$15 \mu W$} \\
\hline Initial battery energy & \multicolumn{2}{|c|}{ 4-14 J } \\
\hline
\end{tabular}

according to the network traffic, because when the traffic load increases especially the intermediate nodes will spend more energy to transmit messages. Therefore, the initial energy of devices is varied linearly from 4 to $14 \mathrm{~J}$ according to the traffic load level. So, when there is low traffic load (10 source nodes) we set the initial energy to $4 \mathrm{~J}$, while if there is high traffic load (100 source nodes) we assign to nodes $14 \mathrm{~J}$. However, the PAN coordinator maintains the same initial energy $(100 \mathrm{~J})$ for all traffic levels, because it is not suffering energy constraints (it may be mains powered).

\subsection{Results and performance analysis}

As presented previously, $\alpha$ is the multiplicative factor that defines any application's needs in terms of prioritized messages latency or energy resources management. In our study, we consider the system behavior for specifying the optimum combination that gives the best results, while keeping a good level of PDR compared to that of the standard.

At the first time, we will show the difference between our algorithm, for different values of the

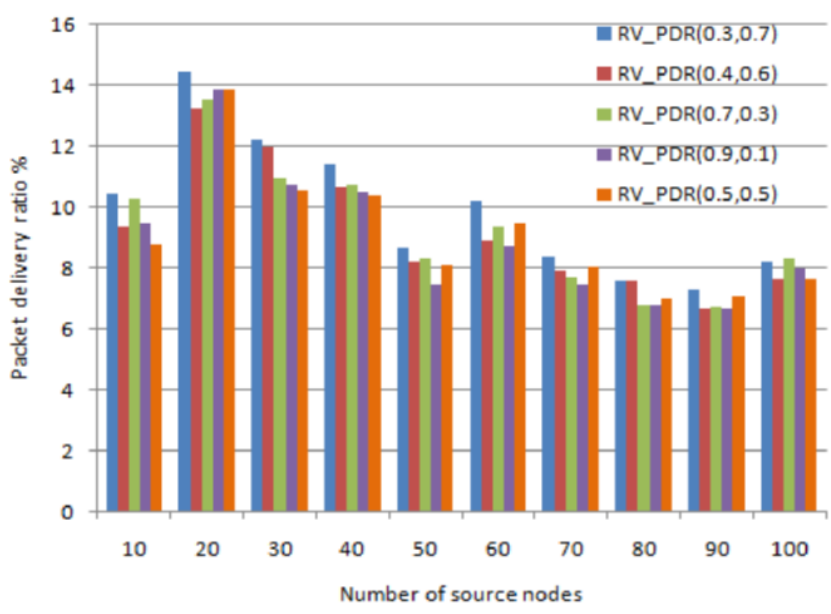

Figure. 4 Packet delivery ratio variation of different $\alpha$ values for BMPriority algorithm

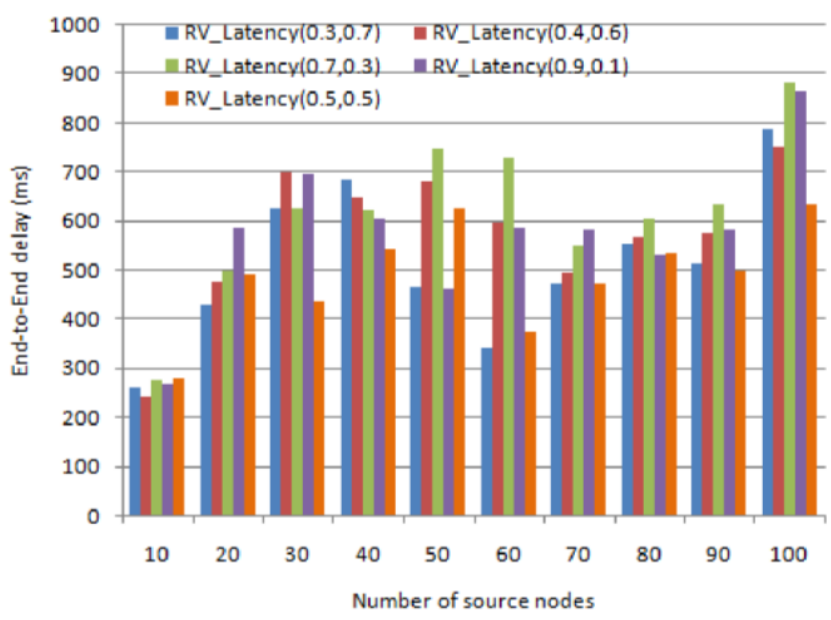

Figure. 5 Prioritized messages latency variation of different $\alpha$ values for BMPriority algorithm

multiplicative factor $(\alpha)$, and the slotted CSMA/CA, in terms of PDR (Fig. 6) and the latency of prioritized messages (Fig. 7), in order to choose the most optimized of them. In both figures, we calculate the relative variation that consists of the difference between the results of our algorithms and those of CSMA/CA algorithm. It refers either an evolution or a diminution of the performances of BMPrioritybased CSMA/CA algorithm compared to CSMA/CA algorithm. After that, we compare our scheme results (with specific combinations) with those of other protocols, in terms of PDR, latency of prioritized messages and of all messages, the throughput, and the number of messages sent over the critical threshold. In Fig. 4 and 5, we consider:

$$
\begin{aligned}
R V_{-} P D R_{(\alpha, 1-\alpha)}= & V_{B M P r i o r i t y-b a s e d} \operatorname{CSMA} / C A(\alpha, 1-\alpha) \\
& -V_{\text {IEEE } 802.14 .5}
\end{aligned}
$$




$$
\begin{aligned}
& R V \_ \text {Latency } \\
&(\alpha, 1-\alpha) \\
&= V_{I E E E} 802.14 .5 \\
&-V_{B M P r i o r i t y-b a s e d} \operatorname{CSMA} / \operatorname{CA}(\alpha, 1-\alpha)
\end{aligned}
$$

Where $R V_{-} P D R$ and $R V_{-} L_{\text {Latency }}$ are the relative variations of our algorithm, in terms of PDR and latency of prioritized messages, respectively.

In terms of latency, it is evident that the values of $\alpha$ that are above 0.5 , will guarantee reduced latency compared to that of the standard. In Fig. 7, we can see that in most traffic levels, it is $\alpha=0.7$ that ensures the best results compared to the other protocols. As well, for the PDR, we note that the results of $\alpha=0.7$ remain better than those of $\alpha=0.9$. In addition; we note in Fig. 6 that $\alpha=0.3$ ensures the highest levels of PDR. Theoretically, this is justified because the values of $\alpha$ that promote the battery energy-level priority give more chance to the intermediate nodes for transmitting messages.

Therefore, that ensures a high throughput, which gives a high level of PDR. In addition, we can remark in both figures (Fig. 6 and 7) that the results of $\alpha=$ 0.5 are neither better than those of $\alpha=0.7$ and $\alpha=$ 0.9 , nor worse than those of $\alpha=0.3$ and $\alpha=0.4$. Because, if we use $\alpha=0.5$, many nodes will obtain the same value of $B E$, although they have different levels of MsgPriority and EnrgPriority. For example, one node has $(1,3)$ and another one has $(3,1)$, both of them will obtain the same value of $B E$ that is 2 . Therefore, that will not promote any one of two priorities, also, that will increase collisions. As result, this value is not beneficial for both priorities. The objective of our algorithm is to verify the compromise between QoS (ensure low latency for prioritized messages) and the good operating of energy resources. According to the results (see Fig.6 and 7), we will compare our algorithm, setting the multiplicative factors on $(0.3,0.7)$ and $(0.7,0.3)$, with the standard at all the performance criteria that we mentioned in the previous subsection. In all simulations presented below, we will use the listed parameters in the Table. 2. In these experiments, we will evaluate the performance of both algorithms according to different traffic levels in the network.

\subsection{Prioritized message latency}

Since our proposed algorithm is based on CSMA/CA of IEEE 802.15.4 standard, we compare its performance to these of IEEE 802.15.4 standard [4] as the baseline, and to these of SMAC [26] and ECA-MAC [25] protocols.

In this experiment, we calculate the average latency of all prioritized messages (MsgPriority $=1$ ) transmitted between the sources and the destinations.

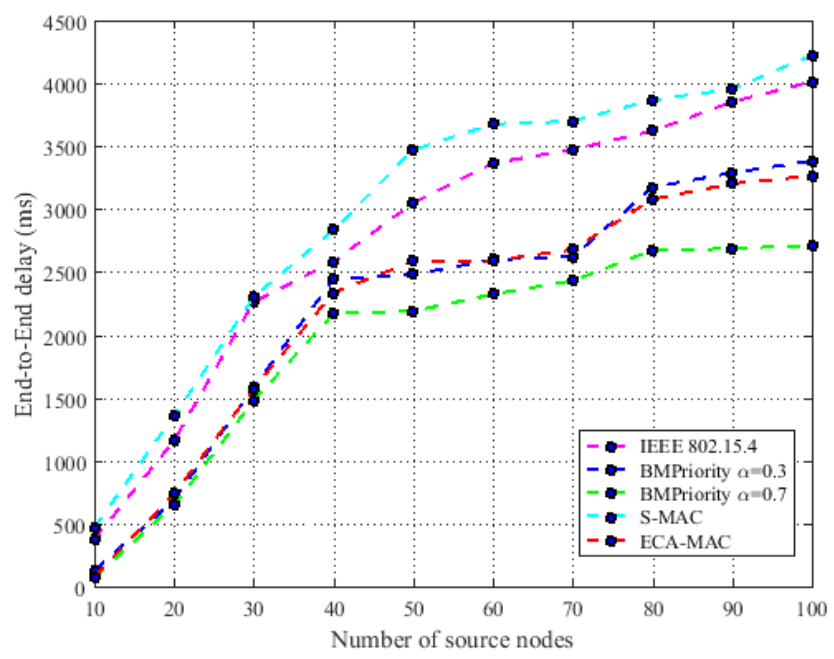

Figure. 6 The average latency of prioritized messages

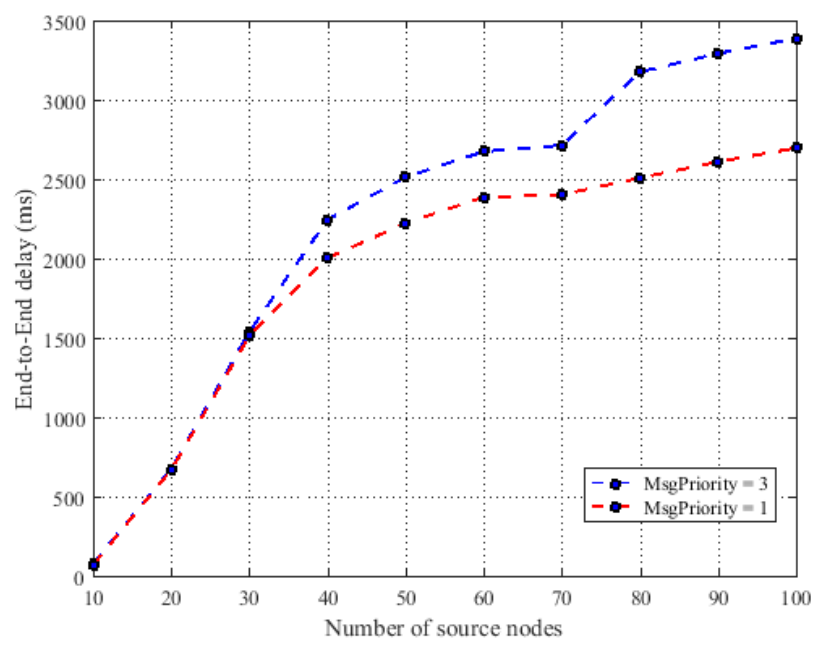

Figure. 7 The average latency of BMPriority-based CSMA/CA algorithm with $\alpha=0.7$ for both priorities MsgPriority $=1$ and MsgPriority $=3$

Fig. 6 shows that in most traffic levels, both values ensure a very low latency compared to S-MAC [26], IEEE 802.15.4 [4], and ECA-MAC protocols [25]. The difference between the results in some experiments is higher than $800 \mathrm{~ms}$ (see Fig. 7). This simulation shows the effectiveness of our algorithm, in terms of latency, against the slotted CSMA/CA algorithm, either in high traffic load (100 source nodes) or in low traffic load (10 source nodes). In this simulation, we note that $\alpha=0.7$ ensures the lowest time latency. Theoretically, this is logical, because this combination provides the highest priority to node that wants to access the medium for sending prioritized messages whatever the energy level of its battery.

In Fig.8, we compare the latency of the lowest MsgPriority and the highest one; and we note that there is a big difference between them, which proves 


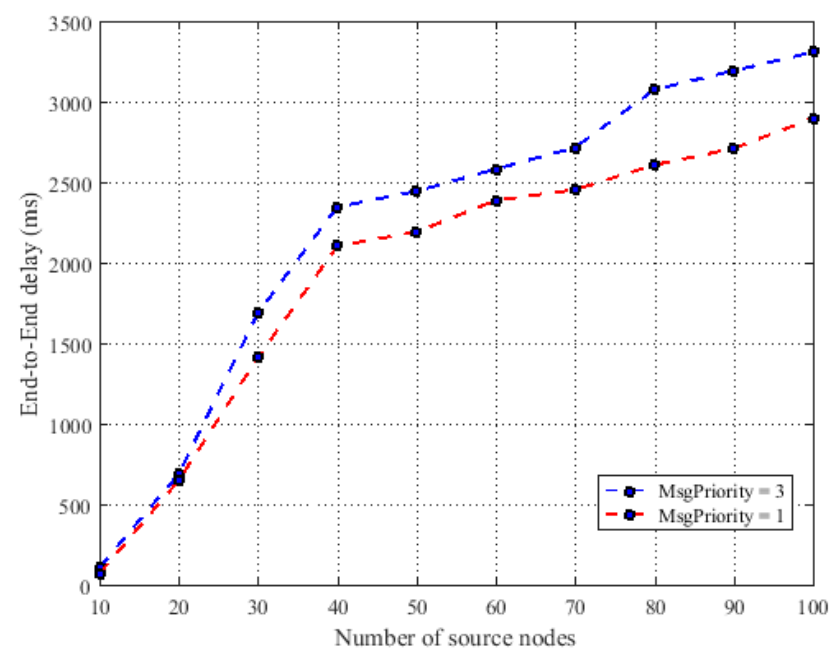

Figure. 8 The latency of BMPriority-based CSMA/CA algorithm with $\alpha=0.3$ for both priorities MsgPriority $=1$ and MsgPriority $=3$

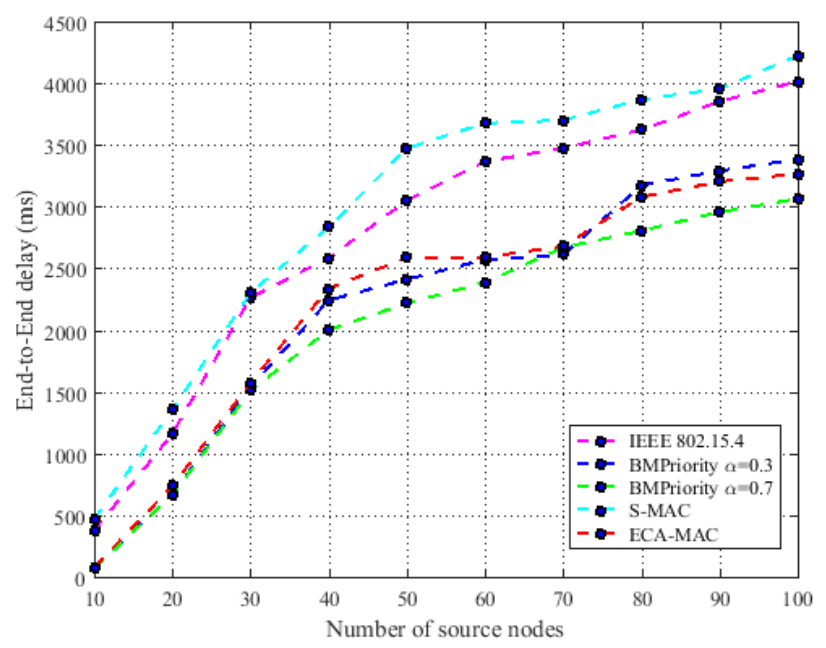

Figure. 9 The average latency vs number of source nodes

the efficiency of our algorithm to reduce the latency of prioritized messages. However, we know that $\alpha=$ 0.3 , in our algorithm, means that the priority given to the battery energy level is more important than the message type, so that is why, in Fig. 8, we can see that there is not a significant difference between the curves.

\subsection{Average network latency}

In this experiment, we calculate the latency of all the sent messages by the source nodes whatever their priorities. We always keep the same environment parameters mentioned in a previous subsection (Table.2).

According to the results shown in Fig. 9, we can confirm that our algorithm, in both cases $\alpha=0.3$ and $\alpha=0.7$, ensures a reduced average latency

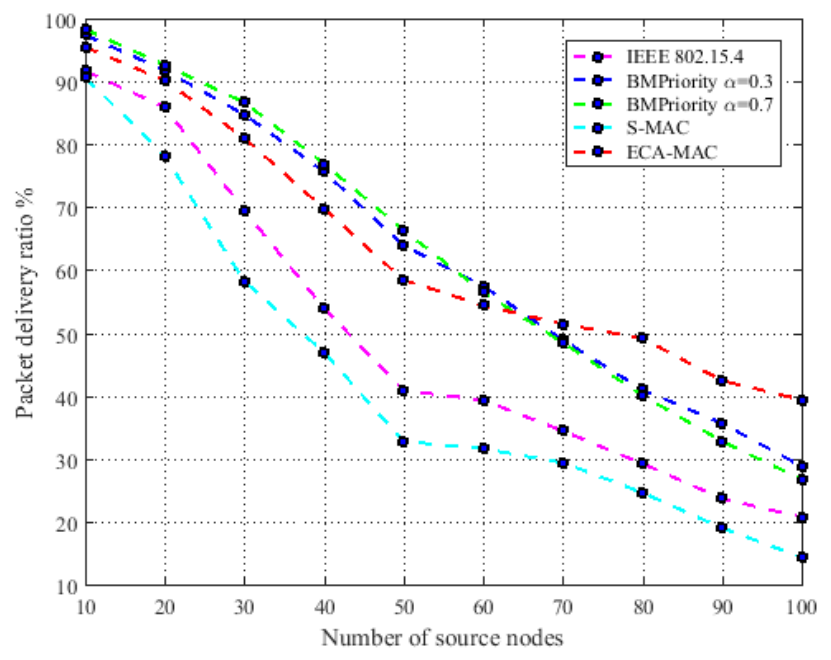

Figure. 10 PDR vs number of source nodes

compared to that provided by the other protocols. We note that $\alpha=0.7$ gives the lowest latency in different traffic levels. In this value, the message priority will ensure, to the intermediate nodes, a high chance to gain the channel contention. So, the medium access priority is respected according to the message priority along the path between the source and the destination node. This will positively affect the average latency of all sent messages in the network. Also, we know that the Wtime depends linearly on $B E$, so that will give the node, in case of collisions, the chance to wait a shorter time, which lead to a low latency.

\subsection{Packet delivery ratio}

In this experiment we evaluate the PDR (that is among of the most considered criteria for evaluating the protocol efficiency) of all transmitted massages in the network, for different traffic levels.

Our algorithm aims to improve the latency and the implication of nodes in the network, without affecting the PDR at all values of $\alpha$.

According to results shown in Fig. 10, we can confirm that our algorithm ensures an improved PDR compared to that of S-MAC, IEEE 802.15.4, and ECA-MAC protocols. In both values, $\alpha=0.3$ and $\alpha=0.7$, the PDR is improved, because our algorithm guarantees a good management of the collision problem, by making different ranges whose the nodes take a random value for the Wtime In our algorithm, MacMinBE belongs to the interval $[2,10]$ and it varies based on the global priority. So, nodes will have different ranges to take the Wtime (see Eq. 3). More the GP is high more the value ofWtime taken by the node is reduced. On the one hand, that gives more chance to nodes with a high GP to obtain medium access faster and to transmit successfully in 


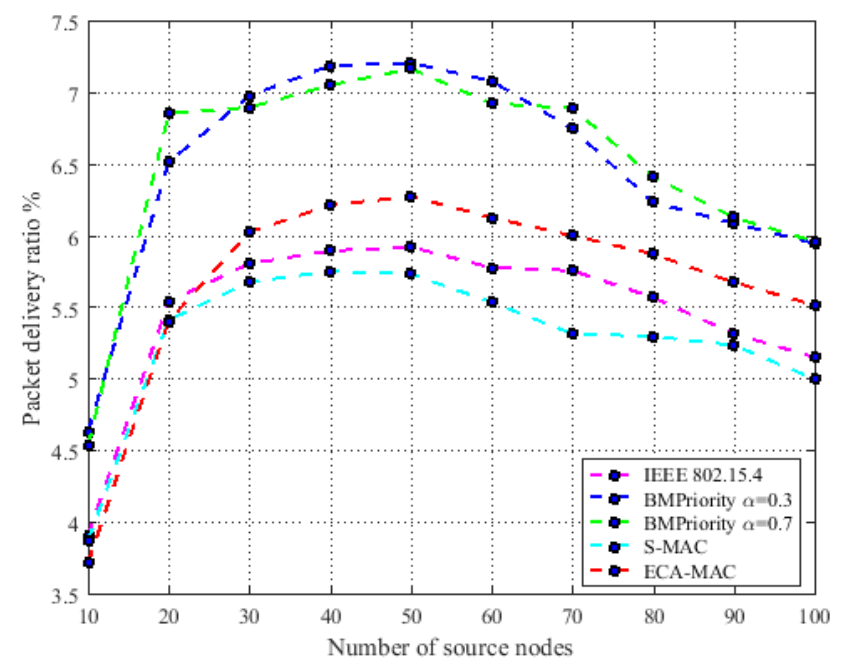

Figure. 11 The throughput vs number of source nodes

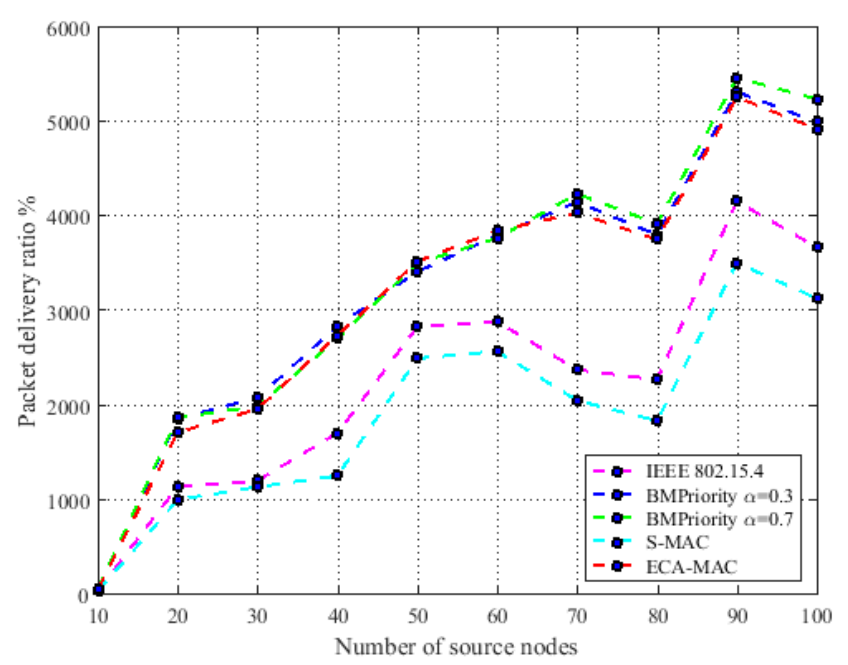

Figure. 12 The numbers of sent messages when the battery energy level is lower than the threshold $S_{1}$

the first attempt, and on the other hand, this scheme allows nodes to avoid collision problem. In Fig. 10, we see that $\alpha=0.3$ gives improved results compared to those of $\alpha=0.7$, because it gives more priority to nodes having a critical battery energy level (especially for the intermediate nodes). As a result, nodes can transmit more data, instead of their battery runs out only waiting for the opportunity to access the medium.

\subsection{Throughput}

In this experiment, we calculate the throughput achieved by all deployed nodes in the network.

Theoretically, the throughput is defined as the amount of data transmitted without error per unit time.

In Fig. 11, we see that both values $\alpha=0.3$ and $\alpha=0.7$ give improved results compared to those of S-MAC, IEEE 802.15.4, and ECA-MAC protocols. However, between these two values, it is $\alpha=0.3$ that gives the best performance. This value allows to nodes that are in critical battery energy level to access the medium and transmit their data.

The nodes that benefit from this process are the intermediate ones. By this way, we offer them a great chance to gain channel access whatever the message priority, which obviously increases the throughput. If an intermediate node battery were exhausted before transmitting its data, the source node would be forced to look for another path and forward the data. This will negatively influence the throughput. However, we remark that above the 70 source nodes, the level of throughput decreases because the high traffic in the network generates more collisions.

\subsection{Node implication in the network}

To evaluate this measure, we calculate the number of sent messages when the node's battery is below the critical threshold, at all nodes deployed in the network.

The purpose of this measurement was to see the impact of the multiplicative factor on the medium access, for nodes being in a critical condition. In Fig.12, we note that the highest results are provided by $\alpha=0.3$, because this last gives to nodes, which theirs batteries in critical state, more chance to access the medium and transmit messages whatever their priorities. Consequently, that gives a good management of energetic resources.

\section{Conclusion}

In this paper, we proposed a new medium access approach called the BMPriority algorithm that based on CSMA/CA technic. BMPriority algorithm considers the priority of nodes in the medium access process by the combination of two priorities; the message priority and the battery energy level. To make weighting between both priorities criteria, we used a multiplicative factor $\alpha$ that can promote one priority over the other. The choice of this multiplicative factor depends on the application's requirement. Through our proposed algorithm we aim, on the one hand, to minimize the end-to-end delay latency for prioritized messages in heterogeneous WSNs. On the other hand, to reduce the waiting delay access to the medium for nodes that have a critical battery level in the network. We proved the effectiveness of the BMPriority algorithm by the obtained results in different simulation scenarios. The shown simulations confirmed that our proposed algorithm achieves an improved packet delivery ratio and low latency of prioritized message that is a logical consequence of the proposed channel access mechanism. In addition, we proved the 
efficiency of our proposed mechanism in other performance criteria (PDR, network latency, and throughput).

The goal of our future research would be an extension of the BMPriority-based CSMA/CA algorithm for improving the latency and extending the lifespan network to be suitable for real-time applications. Then, we intend to adapt it in different network topologies in different application environments.

\section{References}

[1] G. Cerullo, G. Mazzeo, G. Papale, B. Ragucci, and L. Sgaglione, "IoT and Sensor Networks Security", Security and Resilience in Intelligent Data-Centric Systems and Communication Networks, Academic Press, pp. 77-101, 2018.

[2] D. Gopika and R. Panjanathan, "Energy efficient routing protocols for WSN based IoT applications: A review", Materials Today: Proceedings. 2020.

[3] G. Montenegro, N. Kushalnagar, J. Hui, and D. Culler. "Transmission of IPv6 packets over IEEE 802.15.4 networks", Internet proposed standard RFC, Vol. 4944, pp. 130, 2007.

[4] LAN/MAN Standards Committee, Part 15.4: wireless medium access control (MAC) and physical layer (PHY) specifications for low-rate wireless personal area networks (LR-WPANs), IEEE Computer Society, 2003.

[5] G. Anastasi, M. Conti, and M. D. Francesco, "The MAC unreliability problem in IEEE 802.15.4 wireless sensor networks", In: Proc. of the 12th ACM international conference on Modeling, analysis and simulation of wireless and mobile systems, New York, NY, USA, pp. 196-203, 2009.

[6] P. Park, P. D. Marco, P. Soldati, C. Fischione, and K. H. Johansson, "A generalized Markov chain model for effective analysis of slotted IEEE 802.15.4", In: Proc. of 2009 IEEE 6th International Conference on Mobile Adhoc and Sensor Systems, pp. 130-139, 2009.

[7] A. Koubaa, M. Alves, and E. Tovar, "A comprehensive simulation study of slotted CSMA/CA for IEEE 802.15.4 wireless sensor networks", In: Proc. of 2006 IEEE International Workshop on Factory Communication Systems, pp. 183-192, 2006.

[8] X. Liu, C. Leckie, and S. K. Saleem, "Performance evaluation of a converge-cast protocol for IEEE 802.15.4 tree-based networks", In: Proc. of 2010 Sixth International Conference on Intelligent Sensors, Sensor
Networks and Information Processing, pp. 7378, 2010.

[9] J. S. Lee, "An experiment on performance study of IEEE 802.15.4 wireless networks", In: Proc. of 2005 IEEE Conference on Emerging Technologies and Factory Automation, Vol. 2, p. 8, pp. 451-458, 2005.

[10] N. Boughanmi, Y. Song, and E. Rondeau, "Priority and adaptive QoS mechanism for Wireless Networked Control Systems using IEEE 802.15.4", In: Proc. of IECON 2010 - 36th Annual Conference on IEEE Industrial Electronics Society, pp. 2134-2141, 2010.

[11] M. Collotta, G. Scatà, and G. Pau, "A PriorityBased CSMA/CA Mechanism to Support Deadline-Aware Scheduling in Home Automation Applications Using IEEE 802.15.4”, International Journal of Distributed Sensor Networks. Vol. 9, No. 5, pp. 139804, 2013.

[12] L. Zhao, G. Bai, H. Shen, and Z. Tang, "Prioritybased IEEE 802.15.4 CSMA/CA mechanism for WSNs", The Journal of China Universities of Posts and Telecommunications, Vol. 20, No. 1, pp. 47-53, 2013.

[13] G. Ding and R. Farley, "A MAC protocol for wireless personal area networks", In: Proc. of 2013 International Conference on Computing, Networking and Communications (ICNC), IEEE, pp. 900-904, 2013.

[14] E. D. N. Ndih, N. Khaled, and G. D. Micheli, "An Analytical Model for the Contention Access Period of the Slotted IEEE 802.15.4 with Service Differentiation", In: Proc. of 2009 IEEE International Conference on Communications, pp. 1-6, 2009.

[15] I. Dbibih, I. Iala, O. Zytoune, and D. Aboutajdine, "Message Priority CSMA/CA Algorithm for Critical-Time Wireless Sensor Networks", In: Proc. of the Mediterranean Conference on Information \& Communication Technologies 2015. Springer, Cham, pp. 265274, 2016.

[16] A. N. Alvi, S. Khan, M. A. Javed, K. Konstantin, A. O. Almagrabi, A. K. Bashir, and R. Nawaz, "OGMAD: Optimal GTS-Allocation Mechanism for Adaptive Data Requirements in IEEE 802.15.4 Based Internet of Things", IEEE Access, Vol. 7, pp. 170629-170639, 2019.

[17] R. Gupta and S. Biswas, "Priority based IEEE 802.15.4 MAC by varying GTS to satisfy heterogeneous traffic in healthcare application", Wireless Networks, Vol. 26, No. 3, pp. 22872304, 2020.

[18] A. K. Subramanian and I. Paramasivam, "PRIN: A Priority-Based Energy Efficient MAC 
Protocol for Wireless Sensor Networks Varying the Sample Inter-Arrival Time", Wireless Personal Communications, Vol. 92, No. 3, pp. 863-881, 2017.

[19] L. Cheng, X. Zhang, and A. G. Bourgeois, "GTS allocation scheme revisited", Electronics Letters, Vol. 43, No. 18, pp. 1005-1006, 2007.

[20] A. Koubâa, M. Alves, E. Tovar, and A. Cunha, "An implicit GTS allocation mechanism in IEEE 802.15.4 for time-sensitive wireless sensor networks: theory and practice", Real-Time Systems, Vol. 39, No. 1, pp. 169-204, 2008.

[21] Y. Huang, A. Pang, and H. Hung, "An Adaptive GTS Allocation Scheme for IEEE 802.15.4", IEEE Transactions on Parallel and Distributed Systems, Vol. 19, No. 5, pp. 641-651, 2008.

[22] "The Network Simulator - ns-2." [Online]. Available: https://www.isi.edu/nsnam/ns/. [Accessed: 12-Dec-2020].

[23] I. Iala, I. Dbibih, O. Zytoune, M. Rziza, and D. Aboutajdine, "A Kalman Filter Process for Energy Optimization in WSNs", Journal of Communications Software and Systems, Vol. 15, No. 1, pp. 9-17, 2019.

[24] I. Iala, I. Dbibih, O. Zytoune, and D. Aboutajdine, "Adaptive Duty-Cycle Scheme Based on a New Prediction Mechanism for Energy Optimization over IEEE 802.15.4 Wireless Network", International Journal of Intelligent Engineering and Systems, Vol. 11, No. 5, pp. 105-113, 2018.

[25] I. Iala, M. Ouadou, D. Aboutajdine, and O. Zytoune, "Energy based collision avoidance at the mac layer for wireless sensor network", In: Proc. of International Conference on Advanced Technologies for Signal and Image Processing (ATSIP), pp. 1-5, 2017.

[26] W. Ye, J. Heidemann, and D. Estrin, "Medium access control with coordinated adaptive sleeping for wireless sensor networks", IEEE/ACM Transactions on networking, Vol. 12, No. 3, p. 493-506, 2004. 